\title{
Emerging therapies for osteoporosis
}

\author{
G. Minisola, A. Iuliano, I. Prevete
}

Azienda Ospedaliera "San Camillo-Forlanini", Ospedale di Alta Specializzazione "San Camillo", Divisione di Reumatologia, Roma, Italy

\section{SUMMARY}

Currently available drugs for the treatment of osteoporosis can still be improved in terms of pharmacokinetics, pharmacodynamics and management. New approaches for the development of innovative drugs are possible thanks to our increasing understanding of the bone tissue biology and the cellular and molecular processes that regulate it. One of the new anti-bone resorption agents, odanacatib, a selective cathepsin-K inhibitor, is in late phase III clinical research. Among new bone anabolic drugs, those that have an action on the Wnt signaling pathway appear to be particularly promising. The development of new compounds for the treatment of osteoporosis represents an excellent example of translational medicine efforts aimed to extend the range of treatment options for osteoporosis, a very common disease with a high social and economic impact, particularly when causing fractures.

Key words: Osteoporosis, Emerging therapies.

\section{INTRODUCTION}

The increase in average life expectancy 1 has meant that the treatment of osteoporosis (OP) remains one of most pressing challenges in modern medicine. The pharmacokinetic, pharmacodynamic and management profiles of currently available drugs in this field undeniably leave room for improvement. It is therefore evident that recent research has focused on the development of new agents that, besides ensuring better efficacy, safety and tolerability, can also offer more convenient treatment plans and dose regimens to enhance compliance too. Given this is a silent disease until a fracture occurs, treatment compliance amongst OP patients is often poor.

Many potential approaches to the development of new drugs that can influence bone remodeling are based on our increasingly detailed knowledge of the bone tissue biology and the cellular and molecular processes that regulate the activity and signaling mechanisms of bone-forming cells named osteoblasts (OBs), cells responsible for bone resorption named osteoclasts (OCs) and their mediators. In addition, some of the new agents can neutralize a Wnt signal- ing pathway, which is particularly important for the osteoblast function.

The improvement in the treatment options expected over the next few years will allow physicians to choose the most appropriate therapy for each patient, according to the therapy personalization criteria, leading to individualized treatment plan for the management of OP.

This review describes the features of future drugs distinguishing them into the two classic categories: agents that inhibit reabsorption and agents that stimulate bone formation.

\section{Bone modeling and remodeling}

Bone modeling is a process that shapes the skeleton during growth. Despite being typical during the bone growth and development phase in the young, it also takes place at a much slower and rather insignificant pace for the rest of human life.

Bone remodeling is the mechanism that ensures bone turnover and bone mass maintenance. Unlike bone modeling, it is based on the interplay between the bone resorption phase and the bone formation phase. Bone remodeling takes place on the surface of the bone and is performed by the various 
basic multicellular units (BMUs), which incorporate both OBs and OCs working in a cyclical and timed manner.

The cell activity inside the BMUs is balanced when the amount of bone formed equals the amount of bone resorbed, whereas remodeling increases in proportion to the number of active BMUs and their activation frequency.

During OP, the loss of bone mass occurs because cell activities in the BMUs are either uncoupled or coupled but unbalanced. The remodeling process involves the osteoclast activation mediated by the receptor activator of the nuclear factor kappa-B ligand (RANKL), bone resorption, replacement of OBs by OCs and the deposition of the bone matrix, which will undergo mineralization. At the end of the formation phase, OBs either die, as a result of apoptosis, or remain incorporated in the matrix as osteocytes. The subsequent remodeling cycle takes place when osteoclastogenesis is re-stimulated by the RANKL expressed by the OBs and, as was recently demonstrated, also by OCs (1).

\section{NEW BONE RESORPTION INHIBITORS}

\section{Odanacatib}

Cathepsins are lysosomal homeostatic proteases of the cysteine protease family that take part in the proteolysis of collagen during bone turnover. Among the eleven different types observed, Cathepsin K (CatK) is the most important for bone remodeling, given its potent collagenase activity.

CatK plays a crucial role in the degradation of the organic bone matrix. During the OC resorption phase, CatK, which accumulated in lysosome vesicles, reaches the extroflexions of the ruffled border and is released into the acid microenvironment of the resorption lacunae, where the collagenolytic activity starts.

CatK expression is down-regulated by estrogens and up-regulated by RANKL, tumor necrosis factor (TNF), parathyroid hormone (PTH), vitamin D and various interleukins.
CatK deficiency causes a rare form of osteochondral dysplasia, known as pycnodysostosis, which is characterized by osteosclerosis, brittle bones, high risk of fracture, delayed growth, acro-osteolysis of the distal phalanges, cleidocranial dysplasia, mandibular hypoplasia and cranial alterations associated with delayed fontanel closure.

Specific inhibitors have been developed to neutralize the detrimental effect of CatK on the bones. They bind CatK's natural substrate and prevent it from binding to its natural site. The ideal inhibitor must imitate the physical, chemical and biological characteristics of CatK and have a highly specific and selective pharmacological profile to prevent it from interfering with the activity of other cathepsins.

Unlike bisphosphonates (BP), which exert their anti-resorption effect by binding with the bone, thus affecting the function and the number of the OCs, CatK inhibitors are small molecules that concentrate in the resorption lacunae and prevent the formation of the substrate-CatK complex, leaving the vitality and the number of OCs unchanged. Although recent research has been conducted on a number of CatK inhibitors, such as L-006235, Relacatib, Balicatib, ONO-5334 and odanacatib (ODA), but only the last one proved to have an adequate efficacy and safety, therefore it is currently at an advanced stage of clinical development.

ODA is a small, non-basic molecule with a high yet reversible CatK-inhibition capacity and a pharmacokinetic profile characterized by a long half-life and oral bioavailability (2).

Preclinical studies based on the response of remodeling markers on ovariectomized animals showed that the administration of ODA inhibits bone resorption without reducing formation and increases bone mineral density and strength like other resorption inhibitors $(3,4)$.

Compared to other currently available resorption inhibitors, one of the differences observed is a particular compartmentalized effect consisting in a significant increase in the formation of periosteal bone 
and cortical thickness and a less relevant formation of trabecular bone. In addition, the maintenance of the number and vitality of the OCs that can be obtained with ODA enables these cells to maintain their specific functional activities, except for the bone resorption activity mediated by the CatK release $(5,6)$. On the basis of experimental evidence, it has also been postulated that CatK neutralization promotes bone formation by increasing the production of sphingosine-1-phosphate of osteoclastic origin (7).

In phase I and II studies in postmenopausal women, this drug proved to be effective, safe and well-tolerated (8-12).

In the extension study of the phase II trial, women who have received oral ODA (50 $\mathrm{mg} /$ week) on a continuous basis showed linear percentage increases in lumbar and proximal femur bone mineral density (BMD) (femoral neck, trochanter and total hip) of 11.9, 9.8, 10.9 and 9.5, respectively compared to baseline. Over the same period, the N-terminal telopeptide, a urinary bone resorption marker, dropped by $67.4 \%$, while bone-specific alkaline phosphatase, a serum bone formation marker, showed only a modest reduction by $15.3 \%$ (13).

Data obtained in phase I and II clinical studies showed that ODA and BPs have different effects on bone turnover. ODA appears to uncouple bone formation and bone resorption, whereas BPs maintains the coupling of two phases. In addition, histomorphometric studies performed on 32 biopsy specimens from women taking part in the phase II study showed that a modest reduction in the bone formation marker did not correspond to bone formation suppression and that the number of OCs does not show any significant change.

In a group of women switching to placebo after 2 years of treatment, a transient increase was observed in bone remodeling markers, along with a reversal of the BMD trend, 12 months after drug discontinuation, thus proving that the pharmacological effect of ODA is reversible.

As far as adverse events are concerned, no significant difference was observed be- tween treated women and those taking the placebo.

One recent randomized controlled trial, which lasted two years and involved 214 postmenopausal women, confirmed that ODA reduces bone resorption and maintains bone formation, whilst increasing areal and volumetric BMD and bone strength measured at the hip and spine (14).

The results of the phase III study on over 16,000 postmenopausal women will have to confirm the favorable results of the phase I and II studies conducted to date and provide information about the reduction of fracture risk.

\section{Src tyrosine kinase inhibitors}

The absence of this kinase, which is expressed by the OCs, led to the development of osteopetrosis in mice. Src tyrosine kinase contributes to OC survival and to the development of the ruffled border on the osteoclastic resorption surface.

Saracatinib (AZD0530), a small molecule that inhibits Src tyrosine kinase, has been evaluated in a phase I study with oral doses increased from 60 to $250 \mathrm{mg}$ in 59 healthy men (15).

Results showed a significant reduction, compared to the placebo, in resorption markers, but not in formation markers, suggesting that the agent reduces osteoclastic resorption.

Studies on Saracatinib are currently restricted to osteosarcoma and bone metastases.

\section{Glucagon-like peptide 2}

The glucagon-like peptide 2 (GLP-2) is a hormone produced by intestinal endocrine cells, which modifies the rate of bone resorption, which is higher at night under fasting conditions.

A phase II study on postmenopausal women with a low BMD showed that GLP-2, when administered subcutaneously in the evening for four months, induces a reduction in serum levels of the bone resorption marker CTX without having any effect on the bone formation marker osteocalcin (16). Moreover, the highest of the three doses tested $(3.2 \mathrm{mg})$ was associated with 
a significant increase in femoral BMD (trochanter and total hip).

The results showed that this hormone is able to uncouple the resorption phase from the formation phase in the bone.

\section{Integrin $\alpha v \beta 3$ antagonists}

$\alpha v \beta 3$ is an integrin present in the OCs; which interacts with the bone matrix proteins allowing OCs to attach to the surface of the bone. This interaction is needed to let the lacunae resorption start and also induces anti-apoptotic signaling, which favors OC survival and activity.

In the only significant study conducted so far, L-000845704 (a small $\alpha v \beta 3$-inhibitor molecule) administered at various doses and according to different dosage regimens to postmenopausal women with osteoporosis showed to have an anti-resorption effect and to significantly increase BMD (17).

Although $\alpha v \beta 3$ is theoretically an ideal target for the treatment of OP, there is currently no on-going research in this direction involving L-000845704, most likely due its poor tolerability (headache, dermatitis, pruritus, rash and urticaria).

\section{Other bone resorption inhibitors}

Future prospects for identification and use of potential bone resorption inhibitors are based primarily on the possibility of intervening on the chemical and molecular mechanisms that promote and govern OC formation, activation and subsequent adhesion to the surface of the bone.

These could offer a number of options, some of which are currently undergoing conceptual investigation, preliminary study, pilot investigation or critical re-assessment. They include:

1) the inhibition of the chloride channel (ClC7) responsible for the acidification of the resorption cavity;

2) the inhibition of the V-ATPase proton pump on the osteoclastic resorption surface;

3) the inhibition of Atp6v0d2, a V-ATPase subunit needed for the electro-chemical optimization of the chloride channel ClC-7.

\section{NEW BONE FORMATION} STIMULANTS

\section{New formulations and parathyroid hor- mone analogues}

For the purpose of improving treatment compliance, researchers are investigating new PTH formulations and alternative methods of administration to those currently available with $\mathrm{PTH}(1-34)$ and $\mathrm{PTH}(1-$ 84) (18).

These include the possibility of administering $\mathrm{PTH}(1-34)$ subcutaneously once a week at a dose of $56.5 \mu \mathrm{g}$, as supported by the results of a study, and the corresponding follow-up phase, on patients with primary OP at a high risk of fractures $(19,20)$. The pharmacokinetics of four different doses $(1,2.5,5$ and $10 \mathrm{mg})$ of an oral preparation, known as PTH134, was investigated in a phase I study. The postmenopausal women who were given 2.5 and $5 \mathrm{mg}$ oral doses showed exposure levels similar to those of PTH(1-34) administered at a daily dose of $20 \mu \mathrm{g}$ subcutaneously, with a similar adverse event incidence (21). A study is currently under way on PTH134 treatment in osteopenic and osteoporotic postmenopausal women to evaluate the effects of the molecule on bone remodeling markers.

Research is more advanced in the field transdermal PTH (1-34) involving the use of a micro-needle patch to release the active agent. In one phase II study on osteoporotic postmenopausal women, after 6 months of treatment, BMD measurements showed that three different daily doses (20, 30 and $40 \mu \mathrm{g}$ ) achieved significant dosedependent increases compared to placebo and PTH(1-34) administered subcutaneously. In addition, the highest dose significantly increased total hip BMD compared to both placebo and PTH(1-34) $(22,23)$.

Despite the encouraging results of the pilot study, the PTH(1-34) nasal spray formulation has not been promptly developed, although the possibility of inhalation is currently being investigated (24).

Among the PTH analogues evaluated, after some encouraging animal studies, only the cyclized form 1-31 (ZT-031) was investigated on osteoporotic postmenopausal 
women in whom daily subcutaneous administration led to significant dose-dependent increases in lumbar and femoral BMD (proximal and total hip). The remodeling marker trend, tolerability and safety profile were very similar to those obtained with PTH(1-34) (25). The results of a recent phase II study comparing PTH(1-31) $5 \mathrm{mg}$ tablets with subcutaneous PTH(1-34) and placebo in osteoporotic postmenopausal women have not yet been published (http://www.clinicaltrials.gov/ct2/show/ NCT01321723?term=pth+1-31\&rank=1).

\section{PTH-related Protein}

$\mathrm{PTH}-$ related Protein $(\mathrm{PTH}-\mathrm{rP})$ protein is an analogue of PTH with which it shares the receptor PTHR1. Unlike PTH, continuous administration stimulates bone formation. It has been postulated that this different behavior can be attributed to the existence of two states of receptor PTHR1 activation, one of which is specifically for PTH and responsible for the continuous secretion of cyclic AMP consequent to the binding, the other specific to PTHrP and responsible for the critical intermittent secretion. This latter condition imitates bone formation effects associated with intermittent PTH administration (26-29). PTHrP is also thought to have a high bone surface mineralization capacity and it would appear to modulate Wnt signaling components like PTH (30). Following the favorable results of preclinical studies, preliminary tests in postmenopausal women seem to show on the basis of turnover markers that PTHrP uncouples bone formation from resorption and causes significant increases in lumbar BMD (31-35).

A phase II study with a daily subcutaneous synthetic analogue of human PTHrP (BA058) in women with postmenopausal OP, after a 6-month treatment, showed a dose-dependent increase in lumbar and femoral BMD (femoral neck and total hip) that is similar or (at the highest of the three doses considered of 20,40 and $80 \mu \mathrm{g}$ a day) superior to that of the comparator PTH(1-34) $(20 \mu \mathrm{g}$ daily via the subcutaneous route), with an almost identical safety and tolerability profile (36).
One very-recent study compared two different daily doses $(40$ and $60 \mu \mathrm{g})$ of PTHrP and showed that the latter, in postmenopausal women with OP, stimulates bone formation in a similar or superior way to PTH(1-34) at the dose of $20 \mu \mathrm{g}$ administered subcutaneously, but with fewer effects on resorption. These results have not yet been published.

Recruitment is underway for a phase III study comparing the effects on the number of new morphometric vertebral fractures of BA-058 at a dose of $80 \mu \mathrm{g}$ a day administered with PTH(1-34) $20 \mu \mathrm{g}$ (both subcutaneously) and placebo.

\section{Calcilytics}

PTH release is governed by specific receptors known as calcium-sensing receptors (CaSR) located on the surface of the cells in the parathyroid glands. When the calcium concentration in the blood is low, CaSR activity is low and PTH is released. The consequent increase in calcaemia activates CaSR and inhibits PTH secretion. This system can be pharmacologically manipulated using calcimimetics, allosteric modulators of CaSR to treat hypercalcaemia and hyperparathyroidism, or with calcilytics to induce PTH secretion.

Calcilytics therefore imitate a state of hypocalcaemia and cause a critical transient release of PTH, regardless of the levels of calcaemia. The anticipated bone-anabolic activity is, therefore, similar to that of PTH(1-34) administered subcutaneously. Pioneering studies on animals have been followed by tests demonstrating that a compound named SB-423562 and its precursor SB-423557 are able to favor a transient release of endogenous PTH in animals and humans (37-39). Similar results were obtained for both animals and humans with another calcilytic called ATF936 (40).

MK-542 is an allosteric antagonist of CaSR that induces favorable pharmacodynamic responses when administered in combination with alendronate to ovariectomized rats, which could lead to the possibility of combining a PTH secretagogue with a conventional bone resorption inhibitor (41). 
The first study with a calcilytic on a large population of postmenopausal women was conducted using an agent called Ronacaleret (42). After 12 months treatment, the results in terms of efficacy and safety were poorer than expected and the clinical development of this compound was therefore discontinued.

The results of a study with various daily doses of MK-5442 have not yet been published, but it demonstrated that in postmenopausal women who received a previous treatment with alendronate for three years the switch to calcilytics triggers a higher increase in BMD than the continuation of the bisphosphonate therapy.

MK-5442 mechanism of action and development prospects are currently being evaluated (43). It should, however, be considered that the ideal calcilytic should have the following requisites: ability to induce the release of an optimum dose of PTH, short half-life and transient receptor activation in order to prevent prolonged and persistent secretion of PTH.

\section{Activin A antagonists}

Activins, which are proteins expressed largely in the extracellular bone matrix, play a key role in the skeletal tissue and belong to the family of signaling molecules that belong to the TGF $\beta / \mathrm{BMP}$ group.

Activin A (Act A), the most common activin in bone tissue, sends a signal using the Smad pathway, binding two separate receptors, I and II, located on the cell membrane. The binding with the type II receptor (Act RIIA) has a high affinity and is recognized as playing an essential role in osteoclastogenesis.

Although the effects of Act A on bone formation are controversial, recent studies in animals showed that Smad signaling inhibition by Act $\mathrm{A}$ has an agonistic effect on osteoblastic differentiation, as it has favorable effects on bone formation, strength and BMD. This would strongly suggest that Act $\mathrm{A}$ is a negative regulator of bone homeostasis.

In some animal models, a fusion protein consisting of the extracellular domain of ACT RIIA fused to the Fc portion of murine $\operatorname{IgG} 2 \mathrm{a}$ was seen to significantly increase BMD, improve the bone microarchitecture, favor bone formation and increase bone strength (44).

ACE-011 (sotarcept), another fusion protein consisting of the extracellular domain of Act RIIA fused to the Fc portion of human $\mathrm{IgG} 1$, exerts its action by binding with Act $\mathrm{A}$ and preventing it from binding with its endogenous receptors.

In one phase I study with increasing subcutaneous doses (from 0.01 to $3.0 \mathrm{mg} / \mathrm{kg}$ ) and intravenous doses (from 0.03 to 0.1 $\mathrm{mg} / \mathrm{kg}$ ), the administration of a single dose of ACE-011 to post-menopausal women caused an elevation in serum levels of bone alkaline phosphatase and a reduction in levels of CTX and TRACP-5b, with a good safety and tolerability profile (45).

ACE-011 can therefore be considered an effective and safe biologic drug, which is potentially able to exert a dual pro-osteoblastogenetic and anti-osteoclastogenetic action.

\section{Peroxisome proliferator-activated recep- tor modulation}

Peroxisome proliferator-activated receptors (PARs) are crucial transcription factors for many aspects of cell activity. With regard to $\mathrm{OP}$, one particularly interesting area of research and development concerns PPAR $\gamma$ and PPAR $\beta / \delta$, which are both involved in osteogenesis and bone turnover $(46,47)$.

PPAR $\gamma$, in particular, plays a key role in directing the differentiation of mesenchymal precursor cells in an adipogenic rather than osteogenic sense, as shown by the increase in bone mass and the number of $\mathrm{Ob}$ in homozygous PPAR $\gamma$-deficient mice (48). A further indirect confirmation of the involvement of PPAR $\gamma$ in the regulation of bone metabolism comes from a clinical experience involving subjects with type 2 diabetes treated with thiazolidinediones. Indeed, the use of these glucose-lowering medications induces bone mass loss mediated by PPAR $\gamma$ activation (49). Experimental models suggest that inhibition of PPAR $\gamma$ activity could represent a promising strategy for the treatment of OP $(50,51)$. 
Lastly, it is important to note recent reports of PPAR $\beta / \delta$ activation-mediated bone effects consisting in the amplification of canonical Wnt signaling in $\mathrm{OB}$, increased expression of osteoprotegerin and an OBmediated reduction in osteoclastogenesis (52).

$\operatorname{PPAR} \gamma$ and $\beta / \delta$ appear therefore to be important targets for an alternative approach to the treatment of OP.

\section{PHARMACOLOGICAL WNT SIGNALING MODULATION}

\section{Wnt signaling and its inhibitors}

Wnt signaling consists of 19 glycoproteins that govern many aspects of cell growth, differentiation, proliferation, function and death. Wnt signaling is particularly involved in the development, homeostasis, modeling and remodeling of the skeleton. It can be considered a microsystem for the transmission of subsequent and consequential messages (pathways) governed by sophisticated intra- and extra-cellular modulator and inhibitor mechanisms. Depending on the ligands and mediators conditioning its activation and regulation, the following signaling pathways are considered: the canonical (or $\beta$-catenin) pathway, planar cell polarity pathway, cGMP/Ca-mediated pathway and the protein-kinase A pathway; the latter three being considered $\beta$-cateninindependent. Although various pathways can coexist and can be activated at the same time, depending on the tissues and cells one pathway is privileged over the others. In the bone in general and in the OBs in particular, the canonical pathway is generally the most involved. In normal conditions and in the absence of Wnt signaling-promotor ligands, $\beta$-catenin is continually degraded and its levels are very low. This occurs because a degradation kinase complex phosphorylates it and makes it available for ubiquitination and subsequent final proteasomal degradation. The kinases making up the degradation complex are glycogenic syntase-kinase-3 $\beta$ (GSK-3 $\beta$ ), which plays the main role, axin, adenomatous polyposis coli (APC) protein and casein-kinase-1.
When Wnt signaling is activated, $\beta$-catenin is not degraded, it accumulates in the cytoplasm and translocates to the nucleus where it interacts with specific transcription factors and promotes the transcriptional response of the signal's terminal target genes.

The cascade of events that allows $\beta$-catenin translocation into the nucleus involves the interaction of the Wnt glycoprotein ligand with the serpentine or frizzled (Fzd) proteins and with co-receptors represented by lipoprotein receptor-related protein (LRP) 5 or 6 . The formation of the Wnt-FzdLRP5/6 complex inhibits the phosphorylation of $\beta$-catenin by virtue of a process involving the Dishevelled (Dsh) intracellular proteins that are phosphorylated and contribute to the degradation of GSK-3 $\beta$.

The canonical pathway is regulated by sophisticated transmembrane modulation and by intra- and extracellular antagonists. These include Kremen-1 protein, Wnt inhibitory factor (Wif-1), secreted frizzled related protein-1 (sFRP-1), DKK-1 protein and sclerostin.

Canonical pathway activation promotes OB differentiation, maturation and proliferation, preventing at the same time both their apoptosis and osteocytes apoptosis too. These characteristics make the canonical Wnt signaling pathway and its modulation particularly interesting with regard to the development of bone-anabolic agents (53). Indeed, it is believed that the use of the endogenous signaling inhibitor antagonists, anti-sclerostin in particular, directly stimulates bone formation by means of a modeling process that is independent of remodeling and its phases, and of the frequency and entity of BMU activation.

\section{Anti-sclerostin antibodies}

Sclerostin is a protein expressed almost exclusively by the osteocytes and downregulates OBs activity. In mice, sclerostin deletion causes an increase in bone mass due to an increased formation of the cortical and trabecular components.

Subjects who are homozygous for recessive mutation with functional loss of the gene coding for sclerostin are affected by 
sclerosteosis or Van Buchem's disease. The first is a rare condition most commonly observed in South Africa and characterized by generalized osteosclerosis, skeletal deformities, cranial nerve compression, elevated intracranial pressure due to excessive cranial bone development, an absence of nails and premature death; whereas the second is a rare disease mostly common in Holland and characterized by endosteal hyperostosis of the long and cranial bones, protruding chin, prominent forehead and facial nerve paralysis. Family members who are heterozygous for the mutation have a normal phenotype, uncompromised life expectancy, increased bone consistency and low risk of fractures.

However sclerostin is an endogenous Wnt signaling inhibitor and, unlike other inhibitors, has an activity restricted to osteoblastic and osteocytic cell lines, therefore its inhibition is likely to have no extraskeletal effects that could occur when antagonizing other Wnt signaling inhibitors. Sclerostin would therefore appear an optimal target for OP therapy; moreover, all agents able to counteract sclerostin would only exert their action in the areas of the skeleton where it is produced. This would limit the effect to the areas in which the therapeutic intervention is required. In ovariectomized female rats, the administration of anti-sclerostin antibodies blocks and reverses bone mass loss, whereas in adult males it causes an increase in bone formation and mass associated with greater long bone and vertebral strength (54). Similar results have been obtained with animal models of immobilization OP and fracture healing (55).

In 2011, results were published of a phase I study involving single subcutaneous administrations of increasing doses of between 0.1 and $10 \mathrm{mg} / \mathrm{kg}$ of humanized monoclonal antibody (AMG785 - romosozumab) versus sclerostin in male subjects and in postmenopausal women (56). One month after the injection of $10 \mathrm{mg} / \mathrm{kg}$, bone formation markers were elevated to levels similar to those achievable with daily subcutaneous injections of PTH(1-34) for six months, while, at the same time, the reabsorption markers dropped. The significant effect of AMG785 on these markers is most likely due to the potential increase in osteoprotegerin mediated by the Wnt signaling activation. Increases in total femoral and lumbar BMD were similar to or greater than those achieved with PTH(1-34).

The results of a phase II study comparing anti-sclerostin human monoclonal antibody AMG785/CDP7851 and placebo in postmenopausal women with low BMD were recently published (www.amgen. com). According to the protocol, a monthly subcutaneous administration of 70, 140 and $210 \mathrm{mg}$ and three-monthly administrations of 140 and $210 \mathrm{mg}$ were performed. Twelve months after baseline measurements, a consistent increase was observed in lumbar BMD compared to PTH(1-34) and placebo; the most frequent adverse event was an injection site reaction. Two phase III studies are currently under way in postmenopausal women to evaluate the drug's efficacy in preventing fractures.

AMG 167 is another monoclonal antisclerostin antibody currently undergoing phase I trials in men and postmenopausal women with low BMD and who have a negative history for skeletal fragility.

Recently, the phase 1 trials results for Blosozumab (LY2541546), a monoclonal antibody targeting sclerostin, have been shown (57). A previous preclinical study demonstrated that the administration of Blosozumab promoted bone formation and resulted in improvements in bone microstructure and strength (58). These two clinical studies were conducted to assess the safety, tolerability, pharmacokinetics, and pharmacodynamics of Blosozumab in postmenopausal women. The drug exhibited an anabolic effect on bone, was generally well-tolerated and demonstrated an overall favorable safety profile. A phase II trial has been recently completed and preliminary confirming results have already been published (http://www.bone-abstracts. org/ba/0001/ba0001OC5.3.htm).

According to the mechanism of action, not only could anti-sclerostin antibodies be used in OP, but also for bone repair purposes and in conditions with a low turnover, when an increase in bone mass is required. 


\section{Dickkopf-1 antagonists}

Dickkopf-1 (DKK-1), an endogenous inhibitor of Wnt signaling expressed in many tissues, plays a crucial role in bone mass regulation, has been proven in animal models where overexpression or deletion are associated to a severe loss of or increase in bone mass, respectively. DKK-1 neutralization protects rats overexpressing TNF from cytokine-related bone damage and ovariectomized rats from loss of bone mass and mechanical competence (59).

Particularly high DKK-1 levels have been measured in patients with low BMD and postmenopausal women with OP $(60,61)$. In male subjects, DKK-1-inhibition was seen to counter the bone damage associated with rheumatoid arthritis and use of steroids, which induce overexpression of both DKK-1 and SFRP-1 in osteoblastic cell lines (62-64).

This information was at the basis of the development of fully human monoclonal antibodies (RH2-18, PF-04840082, RN564 and BHQ880). Their efficacy and safety are currently being studied and evaluated in postmenopausal OP animal models, osteopenic women and healthy men $(65,66)$. Very early results of the animal studies seem to suggest that pharmacological DKK-1 inhibition causes dose-dependent increases in BMD and increases bone formation on the endocortical and trabecular surfaces. The most likely fields for the application of pharmacological DKK-1-inhibition currently appear to be bone lesions caused by multiple myeloma and, most probably, glucocorticoid-induced OP $(67,68)$.

\section{Glycogen synthase kinase-3 inhibition}

Glycogen synthase kinase-3 (GSK-3 $\beta$ ) enzyme is a serine/threonine protein kinase that governs a number of signal transduction cascades, such as, for instance, cell responses to Wnt signaling.

Most studies on the efficacy of GSK- $3 \beta$ inhibition are based on and experiments.

One study on ovariectomized rats showed the favorable effect of inhibitor LY603281 on the bone. GSK-3 $\beta$ inhibition can be implemented by specific small molecules, such as 603281-31-8, which in ovariectomized rats elevates bone formation marker levels, BMD and bone strength (69). One and animal study showed favorable effects on steroid-induced OP when GSK-3 $\beta$ was inhibited using 6-bromoindirubin-3'-oxime (70). Lastly, an inhibitor called AR28 has shown to increase bone mass in mice after two weeks of treatment (71).

Despite being a potential valid strategy for countering bone loss, GSK-3 $\beta$ control does not currently appear to be a promising opportunity for the treatment of OP.

\section{Food for thought}

Pharmacological Wnt signaling modulation using signaling agonists and inhibitor antagonists is an area of great interest and presents an innovative approach for the development of bone anabolics.

However, because of the ubiquity of the various ligands involved in signaling, it is important to consider the risk of adverse events, including those involving normal and neoplastic cell pro-proliferation, including those in extra-skeletal tissues.

Cancer risk, which is higher for the liver and bowel, would appear to be minimal or absent when the bone highly specific inhibitors - such as sclerostin and DKK-1 - are antagonized, and would appear to be higher, in terms of osteosarcoma, in the case of Wif-1 inhibition.

Since it seems that low Wnt signaling is closely associated with OP, normal Wnt signaling contributes to normal bone homeostasis, high Wnt signaling increases bone mass and, when particularly high, favors bone proliferation, it is essential to identify with precision the duration and type of treatment and the therapeutic range of agents that interfere with signal activation and transmission, giving priority to those with particular bone cell specificity. Lastly, it is important to remember that signal activation can favor the development of vascular calcification and promote exuberant osteophyte formation.

\section{Other bone formation stimulants}

There are many anabolic agents and treatment strategies that can contribute to the 
formation of the bone and its mass. Among potential ways of manipulating Wnt signaling, one apparently interesting option involves the neutralization of SFRP-1, signal inhibitor proteins, and the use of lithium, a nonspecific GSK-3 $\beta$ inhibitor. This last approach is supported by a number of studies in animals and humans showing a positive effect on fracture risk.

Other approaches, such as those involving the synthesis of small molecules that imitate Wnt signaling by stimulating its receptor in the Fzd proteins, the development of agents that interfere with the activity of Dvl proteins and the synthesis of histone-deacetylase (HDAC) inhibitors, that inhibits the post-translational acetylation modification of $\beta$-catenin when it migrates into the nucleus, do not currently appear feasible.

Promising therapeutic developments may also come from agents that imitate the activity of bone morphogenetic proteins (BMP) or that inhibit their proteasomal degradation. On the basis of preclinical experience, BMPs 2 and 6 would appear to be the best suited to systemic administration. In addition, and studies suggest that the growth hormone $(\mathrm{GH})$ and the insulin-like growth factor 1 (IGF-1) have a dose-dependent stimulating action on $\mathrm{Ob}$.

At present we still appear to be far from using statin-like compounds, agents that release nitric oxide (nitroglycerin, isossorbide mono- and dinitrate, etc.), serotonin produced by duodenal enterochromaffin cells, osteoblastic proteasome inhibitors (bortezomib) and sirtuin 1 activators (resveratrol).

Similar considerations apply to the transforming growth factor $\beta$ (TGF $\beta$ ), osteocalcin modulation and the intervention on transcription factors (Runx2, Wif1, etc.).

\section{CONCLUSIONS}

Old age is associated with unbalanced bone formation, with is the main pathogenic mechanism underlying OP. The agents described above may interfere with this mechanism on various levels and become potentially strategic for an increasingly efficacious treatment of OP and, most importantly, of its most severe form with a high risk of fractures. However, in the longterm, not only will it be necessary to evaluate the efficacy profile, but also their safety and tolerability according to the same standards adopted for the pharmacological options available today.

In addition, studies and observations are required to define the degree of interaction between the new anti-osteoporotic agents and other medications used by the same individual to treat other conditions and to evaluate the possibility and validity of $\mathrm{OP}$ treatments involving sequential or combined use of other new medications or currently available ones.

The future of OP therapy is full of expectations. Doctors and patients need new drugs and hope that new agents will soon be available to meet these expectations. This will make it possible to improve control of this common condition that, particularly when it is severe or associated with fractures, has a significant effect on the quality of life of patients and constitutes a heavy social and economic burden for the community. $\mathrm{OP}$ is a condition for which the prevention and intervention schemes implemented in Italy are still inadequate and unsatisfactory.

\section{REFERENCES}

1. Nakashima T, Hayashi M, Fukunaga T, Kurata $\mathrm{K}$, Oh-Hora $\mathrm{M}$, Feng JQ, et al. Evidence for osteocyte regulation of bone homeostasis through RANKL expression. Nat Med. 2011; 17: 1231-4.

2. Gauthier JY, Chauret N, Cromlish W, Desmarais $\mathrm{S}$, Duong LT, Falgueyret JP, et al. The discovery of odanacatib (MK-0822), a selective inhibitor of cathepsin K. Bioorg Med Chem Lett. 2008; 18: 923-8.

3. Masarachia PJ, Pennypacker BL, Pickarski M, Scott KR, Wesoloswki GA, Smith SY, et al. Odanacatib reduces bone turnover and increases bone mass in the lumbar spine of skeletally mature ovariectomized rhesus monkeys. J Bone Miner Res. 2012; 27: 509-23.

4. Cusick T, Chen CM, Pennypacker BL, Pickarski M, Kimmel DB, Scott BB. Odanacatib treatment increases hip bone mass and cortical thickness by preserving endocortical bone 
formation and stimulating periosteal bone formation in the ovariectomized adult rhesus monkey. J Bone Miner Res. 2012; 27: 524-37.

5. Kong WN. Potential role of odanacatib in the treatment of osteoporosis. Clin Interv Aging. 2012; 12: 235-47.

6. Zerbini CA, McClung MR. Odanacatib in postmenopausal women with low bone mineral density: a review of current clinical evidence. Ther Adv Musculoskelet Dis. 2013; 5: 199-209.

7. Lotinum S, Kiviranta R, Matsubara T, Alzate JA, Neff L, Lüth A, et al. Osteoclast-specific cathepsin $\mathrm{K}$ deletion stimulates S1P-dependent bone formation. J Clin Invest. 2013; 123 : 666-81.

8. Rodan SB, Duong LT. Cathepsin K - a new molecular target for osteoporosis. Bonekey Osteovision. 2008; 5: 16-24.

9. Stoch SA, Zajic S, Stone J, Miller DL, Van Dick K, Gutierrez MJ. Effect of the cathepsin $\mathrm{K}$ inhibitor odanacatib on bone resorption biomarkers in healthly postmenopausal women: two double blind, randomized, placebo-controlled phase I studies. Clin Pharmacol Ther. 2009; 86: 175-82.

10. Bone HG, McClung MR, Roux C, Recker RR, Eisman JA, Verbruggen N. Odanacatib, a cathepsin-K inhibitor for osteoporosis: a two year study in postmenopausal women with loW bone density. J Bone Miner Res. 2010; 25: 937-47.

11. Costa AG, Cusano NE, Silva BC, Cremers S, Bilezikian JP. Cathepsin K: its skeletal actions and role as a therapeutic target in osteoporosis. Nat Rev Rheumatol. 2011; 7: 447-56.

12. Eisman JA, Bone HG, Hosking DJ, McClung MR, Reid IR, Rizzoli R. Odanacatib in the treatment of postmenopausal women with low bone mineral density: three year continued therapy and resolution effect. J Bone Miner Res. 2011; 26: 242-51.

13. Langdahl B, Binkley N, Bone H, Gilchrist N, Resch H, Rodriguez Portales J, et al. Odanacatib in the treatment of postmenopausal women with low bone mineral density: five years of continued therapy in a phase 2 study. J Bone Miner Res. 2012; 27: 2251-8.

14. Brixen K, Chapurlat R, Cheung AM, Keaveny TM, Fuerst T, Engelke K, et al. Bone density, turnover, and estimated strength in postmenopausal women treated with odanacatib: a randomized trial. J Clin Endocrinol Met. 2013; 98: 571-80.

15. Hannon RA, Clack G, Rimmer M, Swaisland A, Lockton JA, Finkelman RD, et al. Effects of the Src kinase inhibitor saracatinib (AZD0530) on bone turnover in healthy men: a randomized, double-blind, placebo-controlled, multiple-ascending-dose phase I trial. J Bone Miner Res. 2010; 25: 463-71.

16. Henriksen DB, Alexandersen P, Hartmann B,
Adrian CL, Byrjalsen I, Bone HG, et al. Fourmonth treatment with GLP-2 significantly increases hip BMD: a randomized, placebocontrolled, dose-ranging study in postmenopausal women with low BMD. Bone. 2009; 45: 833-42.

17. Murphy MG, Cerchio S, Stoch A, Gottesdiener K, Wu M, Recker R. Effect of L000845704, an $\alpha v \beta 3$ integrin antagonist, on markers of bone turnover and bone mineral density in postmenopausal osteoporotic women. J Clin Endocrinol Metab. 2005; 90: 2022-8.

18. Baron R, Hesse E. Update on bone anabolics in osteoporosis treatment: rationale, current status and perspectives. J Clin Endocrinol Metab. 2012; 97: 311-25.

19. Nakamura T, Sugimoto T, Nakano T, Kishimoto H, Ito M, Fukunaga M, et al. Randomized teriparatide [human parathyroid hormone (PTH) 1-34] once-weekly efficacy research (TOWER) trial for examining the reduction in new vertebral fractures in subjects with primary osteoporosis and high fracture risk. J Clin Endocrinol Metab. 2012; 97: 3097-106.

20. Sugimoto T, Shiraki M, Nakano T, Kishimoto $\mathrm{H}$, Ito M, Fukunaga M, et al. Vertebral fracture risk after once weekly teriparatide injections: follow up study of teriparatide once wekly efficacy research (TOWER) trial. Curr Med Res Opin. 2013; 29: 195-203.

21. Hammerle SP, Mindeholm L, Laumìnonen A, Kisese B, Loeffler R, Harfts E, et al. The single dose pharmacokinetic profile of a novel oral human parathyroid hormone formulation in healthy postmenopausal women. Bone. 2012; 50: 965-73.

22. Cosman F, Lane NE, Bolognese MA, Zanchetta JR, Garcia-Hernandez PA, Sees K, et al. Effect of transdermal teriparatide administration on bone mineral density in postmenopausal women. J Clin Endocrinol Metab. 2010; 95: $151-8$.

23. Daddona PE, Matriano JA, Mandema J, Maa YF. Parathyroid hormone (1-34)-coated microneedle patch system: clinical pharmacokinetics and pharmacodynamics for treatment of osteoporosis. Pharm Res. 2011; 28: 159-65.

24. Shoyele SA, Sivadas N, Cryan SA. The effects of excipients and particles engineering on the biophysical stability and aerosol performance of parathyroid hormone (1-34) prepared as a dry powder for inhalation. AAPS Pharm Sci Tech. 2011; 12: 304-11.

25. Nemeth EF. ZT- 031, a cyclized analog of parathyroid hormone (1-31) for potential treatment of osteoporosis. Drugs. 2008; 11: 827-40.

26. Martin TJ. Osteoblast-derived PTHrP is a physiological regulator of bone formation. $\mathrm{J}$ Clin Invest. 2005; 115: 2322-4.

27. Rosenblatt M. When two keys fit one lock, surprises follow. Nat Chem Biol. 2009; 5: 707-8. 
28. Dean T, Vilardaga JP, Potts Jr JT, Gardella TJ. Altered selectivity of parathyroid hormone (PTH) and PTH-related protein (PTHrP) for distinct conformations of the PTH/PTHrP receptor. Mol Endocrinol. 2008; 22: 156-66.

29. Esbrit P, Alcaraz MJ. Current perspectives on parathyroid hormone (PTH) and $\mathrm{PTH}-$ related protein as bone anabolic therapies. Biochem Pharmacol. 2013; 85: 1417-23.

30. Vilardaga JP, Romero G, Friedman PA, Gardella TJ. Molecular basis of parathyroid receptor signaling and trafficking: a family B GPCR paradigm. Cell Mol Life Sci. 2011; 68: 1-13.

31. Kartsogiannis V, Moseley J, McKelvie B, Chou ST, Hards DK, Ng KW, et al. Temporal expression of PTHrP during endochondral bone formation in mouse and intramembranous bone formation in an in vivo rabbit model. Bone. 1997; 21: 385-92.

32. Stewart AF, Mangin M, Wu T, Goumas D, Insogna KL, Burtis WJ, et al. Synthetic human parathyroid hormone-like protein stimulates bone resorption and causes hypercalcemia in rats. J Clin Invest, 1998; 81: 596-600.

33. Plotkin H, Gundberg C, Mitnick M, Stewart AF. Dissociation of bone formation from resorption during 2 week treatment with human parathyroid hormone-related peptide (1-36) in humans: potential as anabolic therapy for osteoporosis. J Clin Endocrinol Metab. 1998; 83: 2786-91.

34. Horwitz MJ, Tedesco MB, Gundberg C, Garcia-Ocana A, Stewart AF. Short-term, highdose parathyroid hormone-related protein as a skeletal anabolic agent for the treatment of postmenopausal osteoporosis. J Clin Endocrinol Metab. 2003; 88: 569-75.

35. Miao D, He B, Jiang Y, Kobayashi T, Sorocéanu MA, Zhao J. Osteoblast-derived PTHrP is a potent endogenous bone anabolic agent that modifies the therapeutic efficacy of administered PTH 1-34. J Clin Invest. 2005; 115 : 2402-11.

36. Lippuner K. The future of osteoporosis treatment - a research update. Swiss Med Wkly. 2012; 142: w13624.

37. Gowen M, Stroup GB, Dodds RA, James IE, Votta BJ, Smith BR, et al. Antagonizing the parathyroid calcium receptor stimulates parathyroid hormone secretion and bone formation in osteopenic rats. J Clin Invest. 2000; 105: 1595-604.

38. Nemeth EF. The search for calcium receptor antagonists (calcilytics). J Mol Endocrinol. 2002; 29: 15-21.

39. Kumar S, Matheny CJ, Hoffman SJ, Marquis RW, Schultz M, Liang X, et al. An orally active calcium-sensing receptor antagonist that transiently increases plasma concentrations of PTH and stimulates bone formation. Bone. 2010; 46: 534-42.

40. John MR, Widler L, Gamse R, Buhl T, Seu- wen K, Breitenstein W, et al. ATF936, a novel oral calcilytic, increases bone mineral density in rats and transiently releases parathyroid hormone in humans. Bone. 2011; 49: 233-41.

41. Fisher JE, Scott K, Wei N, Zhao JZ, Cisick $\mathrm{T}$, Tijerina $\mathrm{M}$, et al. Pharmacodynamic response to combined treatment regimens with the calcium sensing receptor antagonist JTT305/MK-5442 and alendronate in osteopenic ovariectomized rats. Bone. 2012; 50: 1332-42.

42. Fitzpatrick LA, Dabrowski CE, Cicconetti G, Gordon DN, Papapoulos S, Bone HG 3rd, et al. The effects of ronacaleret, a calciumsensing receptor antagonist, on bone mineral density and biochemical markers of bone turnover in postmenopausal women with low bone mineral density. J Clin Endocrinol Metab. 2011; 96: 2441-9.

43. Cabal A, Mehta K, Ross DS, Shrestha RP, Comisar W, Denker A, et al. A semimechanistic model of the time-course of release of PTH into plasma following administration of the calcilytic JTT-305/MK-5442 in humans. J Bone Miner Res. 2013; 28: 1830-6.

44. Fajardo RJ, Manoharan RK, Pearsall RS, Davies MV, Marvell T, Monnell TE, et al. Treatment with a soluble receptor for activin improves bone mass and structure in the axial and appendicular skeleton of female cynomolgus macaques. Bone. 2010; 46: 64-71.

45. Ruckle J, Jacobs M, Kramer W, Pearsall AE, Kumar R, Underwood KW. Single dose, randomized, double-blind, placebo-controlled study of ACE-011(ActRIIA-IgG1) in postmenopausal women. J Bone Miner Res. 2009; 24: 744-52.

46. Davidge Pitts CJ, Kearns A. Update on Medications with adverse skeletal effects. Mayo Clin Proc. 2011; 86: 338- 43.

47. Ahmadian M, Suh JM, Hah N, Liddle C, Atkins AR, Downes $\mathrm{M}$, et al. PPAR $\gamma$ signaling and metabolism: the good, the bad and the future. Nat Med. 2013; 19: 557-66.

48. Akune T. The role of insuline receptor substrates in bone metabolism. Clin Calcium. 2004; 14: 289-92.

49. Lecka-Czernik B. Bone loss in diabetes: use of antidiabetic thiazolidinediones and secondary osteoporosis. Curr Osteoporos Rep. 2010; 8: 178-84.

50. Wan Y, Chong LW, Evans RM. PPAR-gamma regulates osteoclastogenesis in mice. Nat Med 2007; 13: 1496-503.

51. Wei W, Wang X, Yang M, Smith LC, Dechow PC, Sonoda J, et al. PGC1beta mediates PPARgamma activation of osteoclastogenesis and rosiglitazone-induced bone loss. Cell Metab. 2010; 11: 503-16.

52. Scholtysek C, Katzenbeisser J, Fu H, Uderhardt S, Ipseiz N, Stoll C, et al. PPAR $\beta / \delta$ governs Wnt signaling and bone turnover. Nat Med. 2013; 19: 608-13. 
53. Canalis E. Wnt signaling in osteoporosis: mechanisms and novel therapeutic approaches. Nat Rev Endocrinol. 2013 [Epub ahead of print].

54. Li X, Warmington KS, Niu QT, Asuncion FJ, Barrero M, Grisanti M, et al. Inhibition of sclerostin by monoclonal antibody increases bone formation, bone mass, and bone strenght in aged male rats. J Bone Miner Res. 2010; 25 : 2647-56.

55. Ominsky MS, Li C, Li X, Tan HL, Lee E, Barrero M, et al. Inhibition of sclerostin by monoclonal antibody enhances bone healing and improves bone density and strenght of nonfractured bones. J Bone Miner. 2011; 26: 1012-21.

56. Padhi D, Jang G, Stouch B, Fang L, Posvar E. Single dose, placebo controlled, randomized study of AMG785, a sclerostin monoclonal antibody. J Bone Miner Res. 2011; 26: 19-26.

57. McColm J, Hu L, Womack T, Tang CC, Chiang Ay. Single and multiple dose randomized studies of blosozumab, a monoclonal antibody against sclerostin, in healthy postmenopausal women. J Bone Res. 2013 [Epub ahead of print].

58. Ma Y, Page T, Zeng Q, Adrian MD, Halladay D, Yang $X$, et al. Blosozumab, a humanized monoclonal antibody, and a chimeric rodent monoclonal antibody against sclerostin robustly increase bone formation activity in intact monkeys and ovariectomized rats. J Bone Miner Res. 2012; 27: 1062.

59. Heiland GR, Zwerina K, Baum W, Kireva T, Distler JH, Grisanti M, et al. Neutralisation of Dkk-1 protects from systemic bone loss during inflammation and reduces sclerostin expression. Ann Rheum Dis. 2010; 69: 2152-9.

60. Anastasilakis AD, Polyzos SA, Avramidis A, Toulis KA, Papatheodorou A, Terpos E. The effect of teriparatide on serum Dickkopf-1 levels in postmenopausal women with established osteoporosis. Clin Endocrinol. 2010; 72: 752-7.

61. Butler JS, Murray DW, Hurson CJ, O'Brien J, Doran PP, O'Bryne JM. The role of DKK1 in bone mass regulation: correlating serum Dkk1 expression with bone mineral density. J Orthop Res. 2011; 29: 414-8.

62. Wang FS, Lin CL, Chen YJ, Wang CJ, Yang KD, Huang YT, et al. Secreted frizzled-related protein 1 modulates glucocorticoid attenua- tion of osteogenic activities and bone mass. Endocrinology. 2005; 146: 2415-23.

63. Diarra D, Stolina M, Polzer K, Zwerina J, Ominsky MS, Dwyer D. Dickkopf-1 is a master regulator of joint remodeling. Nat Med. 2007; 13: 156-63.

64. Wang FS, Ko J, Yeh DW, Ke HC, Wu HL. Modulation of Dickkopf-1 attenuates glucocorticoid induction of osteoblast apoptosis, adipocytic differentiation and bone mass loss. Endocrinology. 2008; 149: 1793-801.

65. Yaccoby S, Ling W, Zhan F, Walker R, Barlogie B, Shaughnessy JD. Antibody-based inhibition of DKK1 suppresses tumor-induced bone resorption and multiple myeloma growth in vivo. Blood. 2007; 109: 2106-11.

66. Betts AM, Clark TH, Yang J, Treadway JL, Li M, Giovanelli MA, et al. The application of target information and preclinical pharmacokinetic/pharmacodynamic modeling in predicting clinical doses of a Dickkopf-1 antibody for osteoporosis. J Pharmacol Exp Ther. 2010; 1: 2-13.

67. Canalis E, Mazziotti G, Giustina A, Bilezikian JP. Glucocorticoid induced osteoporosis: a pathophysiology and therapy. Osteoporos Int. 2007; 18: 1319-28.

68. Fulciniti M, Tassone P, Hideshima T, Vallet S, Nanjappa P, Ettenberg SA, et al. Anti-DKK1 $\mathrm{mAb}$ (BHQ880) as a potential therapeutic agent for multiple myeloma. Blood. 2009; 114: 371-9.

69. Kulkarni NH, Onyia JE, Zeng Q, Tian X, Liu M, Halladay DL, et al. Orally bioavailable GSK-3alpha/beta dual inhibitor increases markers of cellular differentiation in vitro and bone mass in vivo. J Bone Miner Res. 2006; 21: 910-20.

70. Wang FS, Ko JY, Weng LH, Yeh DW, Ke HJ, $\mathrm{Wu}$ SL. Inhibition of glycogen synthase kinase $3 \beta$ attenuates glucocorticoid induced bone loss. Life Sci. 2009; 85: 685-92.

71. Gambardella A, Nagaraju CK, O'Shea PJ, Mohanty ST, Kottam L, Pilling J, et al. Glycogen synthase kinase- $3 \alpha / \beta$ inhibition promotes in vivo amplification of endogenous mesenchymal progenitors with osteogenic and adipogenic potential and their differentiation to the osteogenic lineage. J Bone Miner Res. 2011; 26: 811-21. 